\title{
Calorimetry of students' heart rate during exercises of various intensity
}

\author{
Roman Nagovitsyn ${ }^{1 *}$, Elena Chelnokova ${ }^{2}$, Olga Vaganova $^{2}$, Zhanna Smirnova $^{2}$, and Maxim \\ Kutepov $^{2}$ \\ ${ }^{1}$ Glazov State Pedagogical Institute, 427621, Glazov, Russia \\ ${ }^{2}$ Minin Nizhny Novgorod State Pedagogical University, 603005, Nizhny Novgorod, Russia
}

\begin{abstract}
The purpose of the study: based on data analysis, to develop a formula for calorimetry of students' heart rate during physical activity and experimentally prove the effectiveness of its application in the training process. The study participants $(\mathrm{n}=98)$ were divided by body weight into groups $(n=7)$, regardless of gender and age (20-25 years). Various mobile devices with the function of heart rate calculation and monitoring of kilocalories burning were used in the implementation of control physical activities at different levels of intensity. Analysis of the obtained calorimetric data for each group and the level of training intensity allowed us to identify patterns and develop a formula based on them for an affordable and simple calculation of kilocalories. $\mathrm{N}=0.00168-0.098 / \mathrm{P}$, where $\mathrm{N}$ is the number of kilocalories burned for 1 heart contraction per 1 kilogram of body weight, $\mathrm{P}$ is the heart rate in physical training (beats per minute). An experimental study confirmed the effectiveness of using the author's formula of heart rate calorimetry for the accuracy and uniformity of kilocalories burning in students when they exercise aerobic physical activity.
\end{abstract}

\section{Introduction}

Today, the market of mobile services in the field of fitness and physical education offers various traditional and innovative systems for monitoring and self-monitoring of students physical performance state $[1,2]$. various functional tests for monitoring all body systems are Popularized [3-5], in particular, the key one for analyzing the fitness of the body - the cardiovascular system $[6,7]$. As shown by many experimental studies $[8,9]$, one of the main conditions for the full functioning of the human cardiovascular system is the systematic physical exercise of an aerobic nature $[10,11]$. It is the systematic, stable, and uniform performance of the aerobic motor activity in training sessions and in General in daily and weekly modes that ensures the transition of the body urgent adaptive responses to loads long-term adaptation [12-14]. Violation of the regularity principle and uniformity in the course of training may not only give the desired health-improving effect, but also leads to serious health disorders $[15,16]$. Only a strictly individual-differentiated approach to the implementation of aerobic physical activity on the basis of systematic self-analysis of

\footnotetext{
*Corresponding author: gto18@mail.ru
} 
energy exchange through the account of consumed and allocated body calories will ensure physical and health success [17].

Monitoring of kilocalories consumed and released by the body is based on calorimetry when fixing the heart rate during exercise $[18,19]$. Assessment of energy exchange in the conditions of physical exercise based on heart rate monitoring is a popular, convenient, relatively inexpensive, non-invasive and universal method [20]. Using fitness trackers with the function of calculating heart rate, which have recently become particularly popular, it is possible to implement objective mobile self-analysis [21-24]. Despite the simplicity of using this method, the dependence of heart rate and kilocalorie consumption during aerobic exercise in various energy modes is not sufficiently studied $[7,25]$. In this regard, the research set a goal: based on data analysis, to develop a formula for calorimetry of students ' heart rate during physical activity and experimentally prove the effectiveness of its application in the training process.

\section{Materials and methods}

\section{Participants}

Experimental work was carried out on the basis of the Glazovsky state pedagogical Institute named after V. G. Korolenko, Russia. The research involved 98 full-time and parttime students of the Institute from 20 to 25 years. For the control part of the study, all students selected for the study were divided into seven identical control groups (KG), 14 people in each group, regardless of gender. The division into groups was implemented by the bodyweight of the subjects: KG1-47 $\pm 2 \mathrm{~kg}, \mathrm{KG} 2-50 \pm 1 \mathrm{~kg}, \mathrm{KG} 3-56 \pm 2 \mathrm{~kg}, \mathrm{KG} 4-60 \pm 1 \mathrm{~kg}$, KG5-66 $\pm 2 \mathrm{~kg}, \mathrm{KG} 6-71 \pm 2 \mathrm{~kg}, \mathrm{KG} 7-78 \pm 2 \mathrm{~kg}$. For the experimental part of the work, two experimental groups were selected from the same students by random sampling: EG1 $(n=32)$ and EG2 $(n=32)$. All students prior to the implementation of the research had the experience of independent and systematic classes with medium and high intensity of aerobic motor activity, at least 3 hours per week.

The procedure of research

The pilot work was carried out for 27 weeks (April - October 2019). The research was conducted in three stages: control (first 10 weeks), intermediate ( 2 weeks) and experimental (last 15 weeks). During the control and experimental stages, at each control session (one running session per week), the research participants performed a control measurement. All respondents completed 3-4 physical exercises per week on their own or under the guidance of an athletics instructor and a teacher of the physical culture Department. In each control session, all participants in the research used mobile devices. They were used to monitor the heart rate and calculate the number of calories burned per session. Control classes of aerobic orientation were chosen by the participants of the experiment independently. The only condition for conducting the experiment was an increase in the average heart rate of the respondents from the first control session to the next by 5 beats per minute.

During the second stage of the research ( 2 weeks), the data obtained for each focus group were analyzed. Based on the implementation of mathematical and statistical processing of the obtained quantitative indicators, the regularities of burning kilocalories in students at different levels of aerobic exercise are revealed. The revealed regularities allowed us to develop the author's formula for calorimetry of students' heart rate during various physical activities using the application and processing of data using the mathematical method of least squares.

At the final experimental stage of the research, during the last 15 weeks of the research, students were given different exercises tasks each time before each control session in running. The task included set time for aerobic exercise (55 \pm 15 minutes) and set amount needed to burn calories during running training (530 \pm 70 calories). Students were required 
to strictly perform the prescribed dosage of aerobic load for a given time. And if possible, burn calories as close as possible to the required numerical value set before training, based on the implementation of a uniform run without using the interval method. EG1 used not only mobile technologies for control measurements but also the author's formula for calorimetry of students' heart rate during physical activity developed at the second stage. In turn, EG2 used only mobile devices for monitoring heart rate and counting the number of calories burned per session when performing control tasks in the implementation of aerobic motor activity.

\section{Results and Discussion}

During the control stage of the study, after each control session, individual data were recorded for the control groups on the average heart rate and the number of calories burned during the training process (60 minutes). The quantitative data obtained during this stage (10 weeks) for the control groups are shown in Table.1:

Table.1. Analysis of the number of calories burnt by students during physical activity of various intensity

\begin{tabular}{|c|c|c|c|c|c|c|c|c|c|c|c|}
\hline Control & 1 & 2 & 3 & 4 & 5 & 6 & 7 & 8 & 9 & 10 & Average \\
\hline Average & $110 \pm$ & $115 \pm$ & $121 \pm$ & $126 \pm$ & $130 \pm$ & $135 \pm$ & $139 \pm$ & $144 \pm$ & 151 & 156 & increase \\
\hline $\begin{array}{l}\text { heart } \\
\text { rate }\end{array}$ & 3 & 2 & 1 & 2 & 2 & 3 & 2 & & \pm 3 & \pm 3 & \\
\hline \multicolumn{12}{|c|}{ CG1 (47 \pm 2$)$} \\
\hline \multirow[t]{2}{*}{$\mathrm{Nh}^{*}$} & $244 \pm$ & $268 \pm$ & $297 \pm$ & $321 \pm$ & $340 \pm$ & $363 \pm$ & $381 \pm$ & $405 \pm$ & 439 & 462 & 21.8 \\
\hline & 4 & 3 & 5 & 4 & 5 & 3 & 2 & 3 & \pm 5 & \pm 4 & \\
\hline \multicolumn{12}{|c|}{$C G 2(50 \pm 1)$} \\
\hline \multirow[t]{2}{*}{$\mathrm{Nh}$} & $261 \pm$ & $286 \pm$ & $316 \pm$ & $340 \pm$ & $361 \pm$ & $386 \pm$ & $407 \pm$ & $430 \pm$ & 467 & 491 & 23 \\
\hline & 6 & & 5 & 4 & 3 & 5 & 3 & 5 & \pm 4 & \pm 6 & \\
\hline \multicolumn{12}{|c|}{$C G 3(56 \pm 2)$} \\
\hline \multirow[t]{2}{*}{$\mathrm{Nh}$} & $292 \pm$ & $320 \pm$ & $354 \pm$ & $382 \pm$ & $406 \pm$ & $434 \pm$ & $455 \pm$ & $484 \pm$ & 523 & 551 & 25,9 \\
\hline & 3 & 5 & 2 & 5 & 7 & 5 & 5 & 4 & \pm 5 & \pm 6 & \\
\hline \multicolumn{12}{|c|}{$C G 4(60 \pm 1)$} \\
\hline \multirow[t]{2}{*}{$\mathrm{Nh}$} & $313 \pm$ & $343 \pm$ & $379 \pm$ & $410 \pm$ & $434 \pm$ & $464 \pm$ & $486 \pm$ & $518 \pm$ & 559 & 591 & 27.8 \\
\hline & 5 & 6 & 3 & 4 & 5 & 4 & 6 & 4 & \pm 5 & \pm 6 & \\
\hline \multicolumn{12}{|c|}{$C G 5(66 \pm 2)$} \\
\hline \multirow[t]{2}{*}{$\mathrm{Nh}$} & $343 \pm$ & $377 \pm$ & $418 \pm$ & $451 \pm$ & $477 \pm$ & $509 \pm$ & $537 \pm$ & $570 \pm$ & 615 & 650 & 30.7 \\
\hline & 4 & 7 & 4 & 5 & 4 & 4 & 6 & 4 & \pm 3 & \pm 6 & \\
\hline \multicolumn{12}{|c|}{ CG6 (71 12$)$} \\
\hline \multirow[t]{2}{*}{$\mathrm{Nh}$} & $370 \pm$ & $406 \pm$ & $449 \pm$ & $483 \pm$ & $513 \pm$ & $549 \pm$ & $577 \pm$ & $613 \pm$ & 662 & 697 & 32.7 \\
\hline & 5 & 4 & 4 & 5 & 6 & 6 & 4 & 4 & \pm 3 & \pm 6 & \\
\hline \multicolumn{12}{|c|}{$C G 7(78 \pm 2)$} \\
\hline \multirow[t]{2}{*}{$\mathrm{Nh}$} & $405 \pm$ & $444 \pm$ & $492 \pm$ & $531 \pm$ & $563 \pm$ & $603 \pm$ & $635 \pm$ & $674 \pm$ & 727 & 768 & 36.3 \\
\hline & 6 & 5 & 3 & 5 & 5 & 4 & 4 & 6 & \pm 4 & \pm 5 & \\
\hline $\begin{array}{l}\text { Average } \\
\text { increase }\end{array}$ & 23 & 25.1 & 27.9 & 30 & 31.9 & 34.3 & 36.3 & 38.4 & $\begin{array}{l}41 . \\
1\end{array}$ & 43.7 & 2.07 \\
\hline
\end{tabular}

The analysis of the obtained data allowed us to conclude that the intensity of the training load, expressed in an increase in heart rate, affects the burning of kilocalories per heart contraction in students. However, when translating the data to 1 kilogram of the 
participant's bodyweight, it was found that the change in the burning of calories does not depend on the body weight of the research participants. Based on these regularities and the method of least squares through mathematical comparison of the individual and average quantitative calorimetric data obtained above for all control groups, the formula was developed: $\mathrm{N}=0.00168-0.098 / \mathrm{P}$, where $\mathrm{N}$ is the number of kilocalories burned for 1 heart contraction per 1 kilogram of body weight, $\mathrm{P}$ is the heart rate during the implementation of aerobic motor activity (beats per minute). This formula takes into account the variation in the level of students heart rate in the implementation of various physical activities, regardless of the subject bodyweight.

After the implementation of the final experimental stage of the study on the implementation of the author's formula, data were obtained on the implementation of the main experimental conditions by focus groups. Namely, when performing the dosage of running on control measurements, burn kilocalories as close as possible to the set mark, running the distance at a uniform pace with a constant heart rate. The average arithmetic values of kilocalories consumption deviation in groups from the specified standard for control tests are shown in Fig. 1:

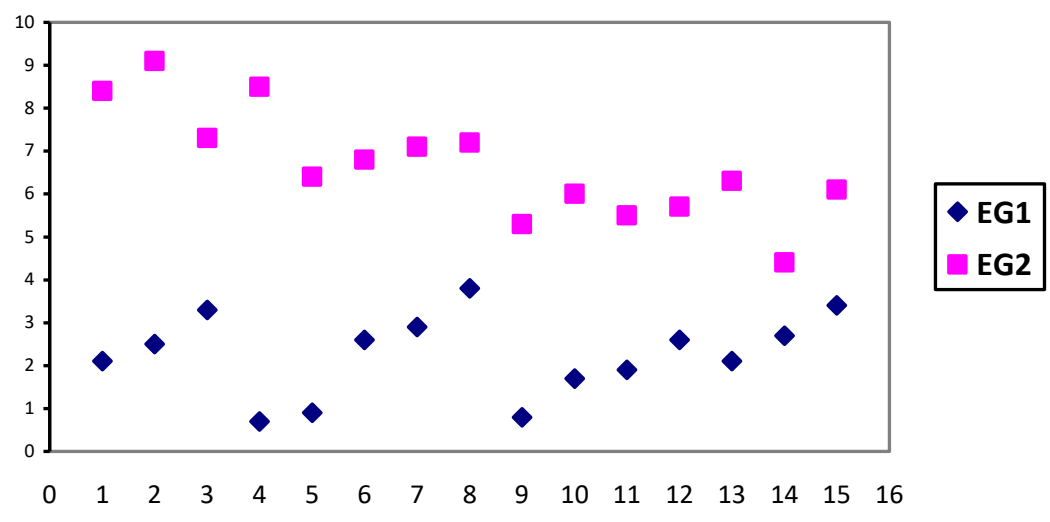

Fig. 1. Arithmetic mean values of kilocalories consumption deviation by groups from the specified standard for control tests

Mathematical and statistical processing of data on the student's T-criterion revealed the reliability of the data difference $(\mathrm{t}=10.7)$ between the arithmetic mean EG1 and EG2 for 15 control measurements for 15 weeks at a significance level of $p<0.01$. Students of EG1, compared with students of EG2, had a statistically significant deviation lower in the burning of calories when implementing control measurements in the run from the numerical parameters set in the study during the experiment.

The obtained mathematical and statistical results of this research are related to the results of research on experimental proof of the increase dependence in kilocalories burning on the intensity of the load, measured based on calculating of athletes heart rate $[3,7]$. Using the introduction of continuous self-monitoring and self-diagnosis technologies, experts reliably determine the effectiveness of various types of motor and energy activity $[9,26]$. The theoretical analysis of studies on the correlation in improving the physical health of the population, in particular the cardiovascular system [17, 19, 27], from an increase in their motor activity during a certain fixed cycle explains the reliability and relevance of the results obtained in our research from a theoretical point of view.

From a practical point of view, the results of this research are consistent with the results of other researches on the experimental effectiveness of the self-monitoring process by non- 
professional athletes using mobile technologies [23, 28]. For example, a research using a special SMART test program [4] or based on the implementation of a smart device for energy expenditure and activity (IDEEA) [20]. With the latest smart device, you can evaluate energy exchange by thirty-five positions and actions that this gadget can identify and record [20]. However, experimental researches show that even the more advanced technology of the IDEA underestimated the burning of calories when walking slowly, but overestimated the speed of walking [20]. Despite the research data, many mobile algorithms have not been confirmed by experimental researches $[1,15]$. An overabundance of proposed mobile applications and guidelines saturates the fitness market with lowquality information and algorithms [21]. Not correctly constructed continuous current monitoring of the athlete's body condition has a negative result on the athlete's indicators $[6,24]$. The society creates a certain distrust of these products and requires simple and affordable methods of the offered services instant verification $[25,29]$. In this direction, the author's development, based on experimental confirmation in this research, allows mobile mathematically reliable and relatively simple diagnostics of the proposed mobile sports and Wellness content. The calculation algorithms proposed in the research will allow users to configure mobile technologies and software according to their preferences and needs.

\section{Conclusions}

The author's research complements previous scientific developments on the implementation of calorimetry in various types of motor activity, including differentiation by the intensity of exercise. The formula " $\mathrm{N}=0.00168-0.098 / \mathrm{P} "$ developed experimentally in the research allows us to calculate mathematically reliably and relatively simply the burning of calories for 1 reduction of the heart per 1 kilogram of body weight of physical exercises participants at various physical loads. An experimental study using mathematical and statistical data processing confirms the effectiveness of the author's formula for calorimetry of the heart rate for the accuracy and uniformity of kilocalories burning in students when they implement the aerobic physical activity.

In the present research, for the first time are experimentally revealed and reliably confirmed patterns of increasing the burning of calories in students at different levels of intensity, depending on their body weight. In this work, the increasing coefficients for increasing the burning of athletes kilocalories ranked by the level of their heart rate during exercise are experimentally justified. This approach is highly relevant in solving one of the youth key problems: increasing the number of students who are overweight and obese. The results of the research offer a number of significant recommendations for optimizing the process of self-monitoring of energy consumption during aerobic exercise.

The authors of the article state that there is no conflict of interest.

\section{Acknowledgements}

The authors would like to sincerely thank the participants who took part in this research for their enthusiastic efforts.

\section{References}

1. I.E. Ioana, C.M. Mihaela, T. Mariana, G. Luminita, C. Constantin, The occupational diary as a smartphone application for monitoring the activities of daily living and physical exertion, Journal of Physical Education and Sport 18, 2008-2013 (2018) 
2. A. Osipov, M. Kudryavtsev, Y. Kopylov, V. Kuzmin, E. Panov, I. Kramida, The possibility of a significant increase in the level of motor activity in students with the use of the potential of computer technology, Physical education of students 22(5), 265-271 (2018)

3. D. Arvidsson, J. Fridolfsson, M. Börjesson, Measurement of physical activity in clinical practice using accelerometers, J Intern Med 286(2), 137-153 (2019)

4. B. Conroy, K.O.Yang, K. Elci, M. Gabriel, J. Styn, A. Wang, S. Kriska, S. Sereika, L. Burke, Physical activity self-monitoring and weight loss: 6-month results of the SMART trial, Med Sci Sports Exerc 43, 1568-1574 (2011)

5. R.S. Nagovitsyn, P.B. Volkov, A.A. Miroshnichenko, Planning of physical load of annual cycle of students', practicing cyclic kinds of sports, training, Physical education of students 21(3), 126133 (2017)

6. T. Jaakkola, S. Yli-Piipari, M. Huhtiniemi, K. Salin, S. Seppälä, H. Hakonen, A. Gråstén, Longitudinal associations among cardiorespiratory and muscular fitness, motor competence and objectively measured physical activity, J Sci Med Sport 22(11), 1243-1248 (2019)

7. W.R. Leonard, Measuring human energy expenditure: what have we learned from the flex-heart rate method? Am J Hum Biol 15(4), 479-489 (2003)

8. G.G. Guimaraes, P.T.V. Farinatti, A.W. Midgley, F. Vasconcellos, P. Vigario, F.A. Cunha, Relationship between percentages of heart rate reserve and oxygen uptake reserve during cycling and running: a validation study, Journal of strength and conditioning research 33(7), 1954-1962 (2019)

9. Y. Wahl, P. Düking, A. Droszez, P. Wahl, J. Mester, Criterion-validity of commercially available physical activity tracker to estimate step count, covered distance and energy expenditure during sports conditions, Front Physiol 8, 725-730 (2017)

10. Z. Kozina, O. Ol'khovyj, V. Temchenko, Influence of information technologies on technical fitness of students in sport-oriented physical education, Physical Education of Students 20(1), 21-28 (2016)

11. R.S. Nagovitsyn, A.Y. Osipov, M.D. Kudryavtsev, K.K. Markov, A.N. Savchuk, L.V. Zakharova, I.I. Orlova, Styles of professional activity of a teacher of higher education of a pedagogical profile, Amazonia Investiga 9(26), 341-348 (2020)

12. R.S. Nagovitsyn, F.H. Zekrin, T.V. Fendel', D.A. Zubkov, Sports selection in martial arts based on the harmonic stability of results at competitions, Journal of Human Sport and Exercise 14(4proc), S867-S876 (2019)

13. R.S. Yagotin, T.V. Degtyarenko, A.I. Bosenko, V.I. Plisko, B.T. Dolinsky, Criterion score of the physical and psychophysiological condition of students in the context of determining their individual adaptability to physical loads, Physical education of students 23(1), 51-57 (2019)

14. Y. Yurchyshyn, Modern information tools: their place in students' extracurriculum and involvement of them in motor activity of health related orientation, Physical Education of Students 19(4), 44-50 (2015)

15. B.A. Lynch, T.K. Kaufman, T.I. Rajjo, K. Mohammed, S. Kumar, M.N. Murad, N.E. Gentile, G.A. Koepp, S.K. McCrady-Spitzer, J.A. Levine, Accuracy of Accelerometers for Measuring Physical Activity and Levels of Sedentary Behavior in Children: A Systematic Review, J Prim Care Community Health 10, 2150132719874252 (2019)

16. R.S. Nagovitsyn, A.V. Tutolmin, Y.G. Maksimov, I.A. Dimova, A.A. Karoyan, D.Y. Skryabina, S.A. Volkov, Motivation for physical activity of people of different ages, Gazzetta Medica Italiana - Archivio per le Scienze Mediche 178(10), 799-806 (2019)

17. B.E. Ainsworth, W.L. Haskell, A.S. Leon, D.R. Jacobs, H.J. Montoye, J.F. Sallis, R.S. Paffenbarger, Compendium of physical activities: classification of energy costs of human physical activities, Med Sci Sports Exerc 25(1), 71-80 (1993)

18. J. Fridolfsson, M. Börjesson, D. Arvidsson, A Biomechanical Re-Examination of Physical Activity Measurement with Accelerometers, Sensors (Basel) 18(10), 3399 (2018) 
19. S.J. Strath, D.R. Bassett, A.M. Swartz, D.L. Thompson, Simultaneous heart rate-motion sensor technique to estimate energy expenditure. Med Sci Sports Exerc 33, 2118-2123 (2001)

20. S. Whybrow, P. Ritz, G.W. Horgan, R.J. Stubbs, An evaluation of the IDEEA activity monitor for estimating energy expenditure, Br J Nutr 109(1), 173-183 (2013)

21. M.D. Chen, C.C. Kuo, C.A. Pellegrini, M.J. Hsu, Accuracy of wristband activity monitors during ambulation and activities, Med Sci Sports Exerc 48(10), 1942-1949 (2016)

22. R.C. Colley, G. Butler, D. Garriguet, S.A. Prince, K.C. Roberts, Comparison of self-reported and accelerometer-measured physical activity in Canadian adults, Health Rep 29(12), 3-15 (2018)

23. A. Henriksen, M.M. Haugen, A.Z. Woldaregay, M. Muzny, G. Hartvigsen, L.A. Hopstock, S. Grimsgaard, Using fitness trackers and smartwatches to measure physical activity in research: analysis of consumer wrist-worn wearables, J Med Internet Res 20(3), e110 (2018)

24. A. Sushames, A. Edwards, F. Thompson, R. McDermott, K. Gebel, Validity and reliability of fitbit flex for step count, moderate to vigorous physical activity and activity energy expenditure. PLoS One 11(9), e0161224 (2016)

25. S. Skender, J. Ose, J. Chang-Claude, M. Paskow, B. Brühmann, E.M. Siegel, K. Steindorf, C.M. Ulrich, Accelerometry and physical activity questionnaires - a systematic review, BMC Public Health 16, 515. (2016)

26. O.I. Vaganova, N.P. Bakharev, J.A. Kulagina, A.V. Lapshova, I.K. Kirillova, Multimedia technologies in vocational education, Amazonia investiga 9(26), 391-398 (2020)

27. L. Podrigalo, H. Artemieva, O. Rovnaya, N. Misevra, Z. Sotnikova-Meleshkina, A. Podavalenko, K. Sokol, I. Robak, Features of physical development and somatotype of girls and women involved in fitness, Pedagogics, psychology, medical-biological problems of physical training and sports 23(4), 189-195 (2019)

28. H. Wennman, A. Pietilä, H. Rissanen, H. Valkeinen, T. Partonen, T. Mäki-Opas, K. Borodulin, Gender, age and socioeconomic variation in 24-hour physical activity by wrist-worn accelerometers: the FinHealth 2017 Survey, Scientific Reports 9(1), 6534 (2019)

29. I.M. Lee, E.J. Shiroma, Using accelerometers to measure physical activity in large-scale epidemiological studies: Issues and challenges. British Journal of Sports Medicine 48(3), 197201 (2014)

30. S.G. Nagovitsyn, Sadiev N.N., Pedagogico-psychological and medico-biological problems of physical culture and sport, 9(1), 84-91 (2014) 